\title{
Episodische Migräne: Was schützt vor der nächsten Attacke?
}

\section{Hier steht eine Anzeige.}

Bei Patienten mit häufigen Migräneattacken wird eine medikamentöse Therapie zur Migräneprophylaxe empfohlen. Hierzu gibt es eine Vielzahl von Substanzen, von denen allerdings nur einige zugelassen sind.

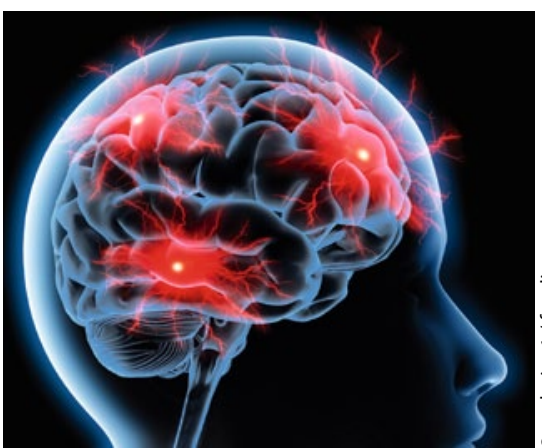

Migräneprophylaxe: Qualitätsmängel bei vielen Studien.
— Die Autoren führten eine systematische Literaturrecherche zur medikamentösen Prophylaxe der episodischen Migräne durch und identifizierten 215 randomisierte Studien. Sie berechneten dann jeweils die 50\%-Responderraten, das heißt die Studien, bei denen die untersuchte Substanz zu einer mehr als 50\%igen $\mathrm{Re}$ duktion der Anfallshäufigkeit führte.

In der Prophylaxe der episodischen Migräne sind metaanalytisch am besten wirksam, die Antikonvulsiva Topiramat und Valproinsäure sowie Betablocker. Die beste Verträglichkeit haben die in dieser Indikation nicht zugelassenen Angiotensin-Rezeptor-Blocker.

- T. A. Shamliyan et al.

Preventive pharmacologic treatments for episodic migraine in adults. J Gen Intern Med. 2013; [Epub ahead of print] DOI: 10.1007/s11606-013-2433-1

\section{Kommentar}

Diese Metaanalyse unterstützt Therapieempfehlungen der Deutschen Migräne- und Kopfschmerzgesellschaft und der Deutschen Gesellschaft für Neurologie. Allerdings wurde Flunarizin, das in Deutschland empfohlen wird, in der Metaanalyse nicht erfasst, da diese Substanz in den USA nicht zugelassen ist. Leider gibt es zu den ACE-Hemmern Captopril und Lisinopril nur jeweils eine Studie, und dasselbe gilt für den Angiontensin-Rezeptor-Blocker Candesartan. Daher kann letztlich nicht mit Sicherheit abgeschätzt werden, ob diese Substanzgruppen in der Migräneprophylaxe wirksam sind. Sie zeichnen sich allerdings durch eine sehr gute Verträglichkeit aus.

Ein echter Wermutstropfen in der Metaanalyse ist die Feststellung, dass die meisten bisher durchgeführten Studien von schlechter Qualität sind, da sie meist zu wenig Patienten einschlossen, schlecht definierte Endpunkte hatten oder die Statistik Mängel aufwies. Streng genommen erfüllen eigentlich nur die Zulassungsstudien zu Topiramat die Anforderungen an ein modernes Studiendesign. 